Draft Version November 25, 2018

Preprint typeset using $\mathrm{LATEX}_{\mathrm{E}}$ style emulateapj v. 6/22/04

\title{
METHANOL IN THE L1551 CIRCUMBINARY TORUS
}

\author{
Glenn J. White ${ }^{1,2}$, C.W.M.Fridlund ${ }^{3}$, P.Bergman ${ }^{4}$, A.Beardsmore ${ }^{5}$, Rene Liseau ${ }^{6}$, M.Price ${ }^{5}$, And R.R.Phillips ${ }^{7}$ \\ Draft version November 25, 2018
}

\begin{abstract}
We report observations of gaseous methanol in an edge-on torus surrounding the young stellar object L1551 IRS5. The peaks in the torus are separated by $\sim 10,000$ AU from L1551 IRS5, and contain $\sim 0.03 M_{\oplus}$ of cold $\mathrm{CH}_{3} \mathrm{OH}$. We infer that the $\mathrm{CH}_{3} \mathrm{OH}$ abundance increases in the outer part of the torus, probably as a result of methanol evaporation from dust grain surfaces heated by the shock luminosity associated with the shocks associated with the jets of an externally located x-ray source. Any methanol released in such a cold environment will rapidly freeze again, spreading $\mathrm{CH}_{3} \mathrm{OH}$ throughout the circumbinary torus to nascent dust grains, planitesimals, and primitive bodies. These observations probe the initial chemical conditions of matter infalling onto the disk.

Subject headings: ISM: general - ISM: individual (L1551, L1551 IRS5, HH 154) - ISM:jets and outflows
\end{abstract}

\section{INTRODUCTION}

In the earliest stages of their formation, low-mass young stellar objects are embedded in flattened gaseous envelopes. Surrounding these nascent protostars, planetary systems, primitive bodies and their attendant dust grains will condense from the infalling material. In this paper, we study the distribution of the organic molecule methanol around a protostellar core, as it is an important constituent of the young material in the disk. The L1551 molecular core (distance $140 \mathrm{pc}$ ) contains a young binary system (seen as an infrared and radio source L1551 IRS5 ) hidden inside a dense envelope providing $\sim 150$ magnitudes of visual extinction (Stocke et al. 1988, White et al. 2000, Fridlund et al.2005). A molecular outflow emanates from the center of the disk (Snell et al. 1980, Kaifu et al. 1984, Fridlund \& White 1989, Rainey et al.1987, Parker et al. 1991), with atomic jet(s) associated with an $\mathrm{x}$-ray emitting region being observed to the SW (Fridlund \& Liseau 1998, Favata et al. 2002, 2003, Bally et al. 2003, Fridlund et al.2005). The protostellar disk is surrounded by a massive (radius $\sim 20,000 \mathrm{AU}$ ) cool envelope that exhibits both rotational and infall motions (Takakuwa et al. 2004, Moriarty-Schieven et al. 2006). Fridlund et al. (2002) used $\mathrm{HCO}^{+}, \mathrm{H}^{13} \mathrm{CO}^{+}$and ${ }^{13} \mathrm{CO} J=1-0$ observations to estimate that the mass of the disk is $\sim$ $2.5 \pm 1.5 M_{\odot}$.

\section{OBSERVATIONS}

Observations of $\mathrm{CH}_{3} \mathrm{OH}\left(96 \mathrm{GHz} 2_{k}-1_{k}\right.$ and $242 \mathrm{GHz}$ $\left.5 k^{-} 4_{k}\right)$, HCN $(J=1-0)$, and CO $(J=1-0)$ were obtained using the Onsala $20 \mathrm{~m}$ and JCMT $15 \mathrm{~m}$ telescopes

1 Department of Physics \& Astronomy, The Open University, Walton Hall, Milton Keynes MK6 7AA, England. g.j.white@open.ac.uk

${ }_{2}$ Space Physics Division, The Rutherford Appleton Laboratory, Chilton, Didcot OX11 0QX, England

3 ESTEC, P.O. Box 299, NL-2200AG Noordwijk, Netherlands. Malcolm.Fridlund@esa.int

4 Onsala Space Observatory, SE-439 92 Onsala, Sweden. bergman@oso.chalmers.se

5 University of Kent, Canterbury CT2 7NR, England. mcp2@star.kent.ac.uk, ab75@star.kent.ac.uk

6 Stockholm Observatory, 10691 Stockholm, Sweden. rene@astro.su.se

7 University of Lethbridge, 4401 University Drive, Lethbridge, Alberta T1K 3M4, Canada.robin.phillips@uleth.ca in Feb 2004 and Jan/Apr 2005. The Onsala observations used an single sideband SIS receiver, with a 1600 channel $\times 25 \mathrm{kHz}$ autocorrelator backend. The JCMT observations of a higher $\mathrm{CH}_{3} \mathrm{OH}$ transition $\left(241 \mathrm{GHz} 5_{k}-4_{k}\right)$ were made using the standard facility $\mathrm{RxA} 2$ receiver. All lines were corrected to a common main beam brightness, $T_{\mathrm{mb}}$ scale. Additional data for $\mathrm{CS}$ and $\mathrm{C}^{34} \mathrm{~S}(J=2-1)$ are taken from Fridlund et al. (2002).

\section{METHANOL}

Methanol plays a key part in the chemistry leading to the production of biogenic molecules, and is an important constituent of icy grain mantles and comets. Thermally excited gas phase $\mathrm{CH}_{3} \mathrm{OH}$ has previously been observed from warm inner envelopes surrounding just a few Class 0 protostars, where it is evaporated from warm dust grains heated by the central protostar, or by an accretion shock at the edge of an infalling envelope (Goldsmith et al. 1999, Velusamy et al. 2002, Maret et al. 2005).

Maps showing the distribution of $\mathrm{CH}_{3} \mathrm{OH}, \mathrm{HCN}$ and CO toward L1551 observed from Onsala are shown in Figure 1, and velocity channel maps for the $96 \mathrm{GHz}$ $\mathrm{CH}_{3} \mathrm{OH}$ and $\mathrm{CS} J=2-1$ lines in Figure 2 .

The methanol has three peaks located $\sim 6500-12000$ AU from IRS5 along a SE-NW line. The high density tracer, $\mathrm{HCN}$ is centrally peaked and marginally resolved, located between the 2 inner methanol peaks. CO emission, which traces low density material, streams orthogonally away to the NE and SW. The methanol appears as an edge-on torus surrounding the $\mathrm{HCN}$ core, and is oriented orthogonally to the CO outflow. The velocity shift between the two $\mathrm{CH}_{3} \mathrm{OH}$ peaks is $0.07 \mathrm{~km} \mathrm{~s}^{-1}$ which if indicative of rotation, would imply a rotational period $\sim 6.5 \times 10^{5}$ yr. Spectra toward L1551 IRS-5 and at the two main methanol peaks are shown in Figure 3, along with the model fits described next.

We have modeled the $\mathrm{CH}_{3} \mathrm{OH}$ line intensities using an accelerated lambda iteration radiative transfer technique for a spherical-core envelope (Phillips \& Little 2000), with an assumed core radius of 5000 AU; envelope radius of $12,500 \mathrm{AU}$; and turbulent velocity of $0.45 \mathrm{~km} \mathrm{~s}^{-1}$ (from the $\mathrm{H}^{13} \mathrm{CO}^{+}$linewidth - Fridlund et al. 2002). The $A$ and $E$ symmetry states of $\mathrm{CH}_{3} \mathrm{OH}$ were calculated separately using collisional rates, energy levels and Einstein 


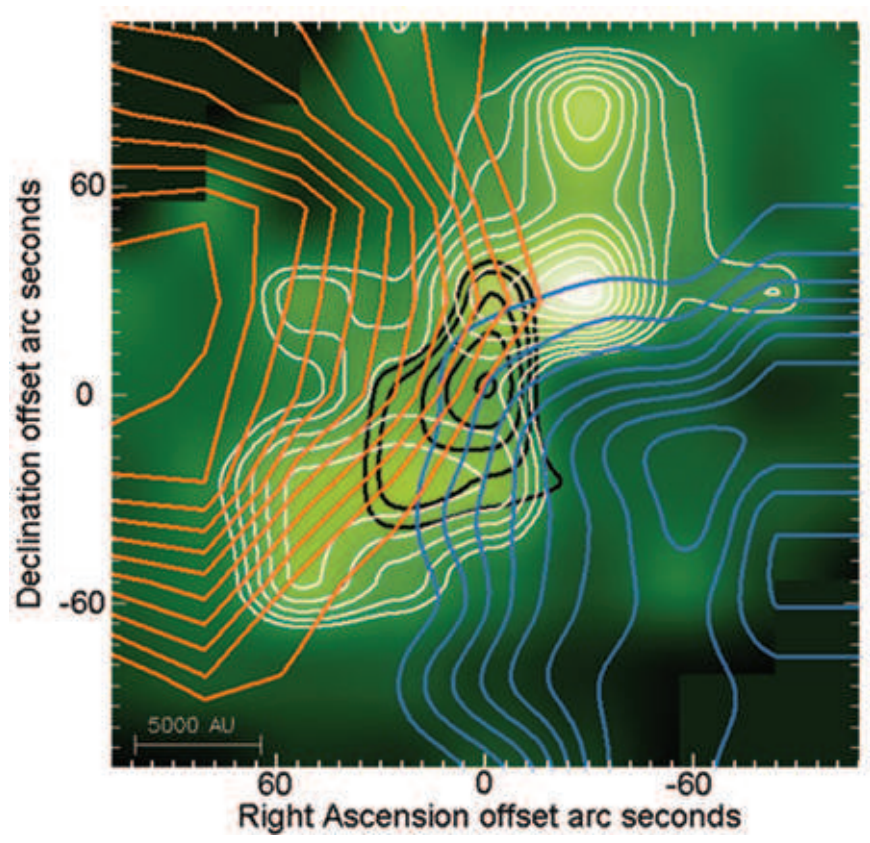

FIG. 1. $-\mathrm{CH}_{3} \mathrm{OH} 22_{0}-1_{0}(A)$ line map (Onsala data at 96.7414 $\mathrm{GHz}$, green image with white contours) superimposed with contours of the $\mathrm{HCN} \mathrm{J}=1-0$ (Onsala data shown as black contours) and (red) and (blue) shifted CO $\mathrm{J}=1-0$ outflows (shown as red and blue contour lines respectively) from the data shown in Fridlund et al. (2002). West is on the left, north at the top. The bottom left bar shows the size scale, for a distance of $140 \mathrm{pc}$.

rates from Müller et al. (2001) and Pottage et al. (2004), and convolved to the Onsala and JCMT beamwidths. A number of models were run spanning a range of temperature, density and abundance gradients to simultaneously match both the relative and absolute spectral line intensities, and the observed morphology of the torus. The observations unfortunately do not provide strong constraints on the $\mathrm{CH}_{3} \mathrm{OH}$ kinetic temperature, and so the temperature and density gradients used in the model were chosen to match those of White et al. 2000 derived from dust continuum measurements.

Using this model the best fit suggests a central hydrogen gas density (at the position of L1551 IRS-5) $n\left(\mathrm{H}_{2}\right)=$ $7.5 \times 10^{5} \mathrm{~cm}^{-3}$ and a methanol abundance $X=8 \times 10^{-10}$ relative to $\mathrm{H}_{2}$. Since the temperature and density are known to decline away from L1551 IRS5 (see Figure 4a, which is derived from White et al.2000), the observed torus structure then requires that the methanol abundance increases to $\sim 4 \times 10^{-9}$ at the inner edge of the methanol ring, and to $10^{-8}$ in the outer envelope, as shown in Figure 4b.

The abundance increase is needed since $\mathrm{H}_{2}$ density is $\sim 20$ times lower at the edge of the $\mathrm{CH}_{3} \mathrm{OH}$ peaks than towards the source centre at L1551 IRS-5. In this best fit model, the torus contains $0.03 M_{\oplus}$ of $\mathrm{CH}_{3} \mathrm{OH}$. Models run with $\mathrm{CH}_{3} \mathrm{OH}$ kinetic temperatures in the torus $\geq 20 \mathrm{~K}$ failed to reproduce the observed disk structure whilst simultaneously matching the absolute and relative line temperatures. Since the gas and dust should be well thermalised at the estimated densities, it is reasonable to assume that the kinetic temperature of the $\mathrm{CH}_{3} \mathrm{OH}$ will be close to the dust temperature (13K from White et al. 2000). The models underestimate the observed
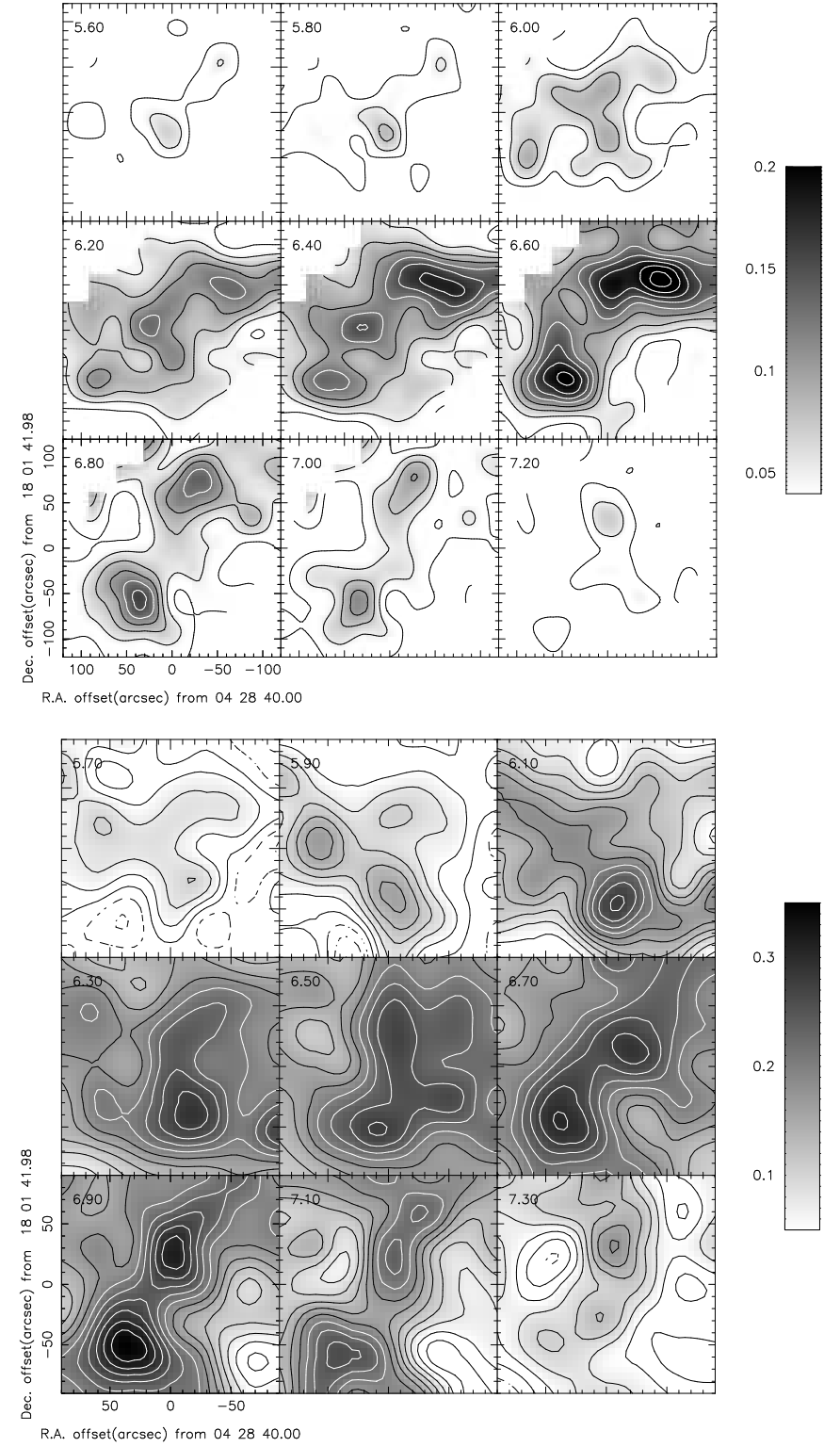

Fig. 2.- [upper] Channel maps in the $2_{0}-1_{0}(A) \mathrm{CH}_{3} \mathrm{OH}$ line. The lowest contour level is $0.15 \mathrm{~K} \mathrm{~km} \mathrm{~s}^{-1}$ and contour intervals are at $0.1 \mathrm{~K} \mathrm{~km} \mathrm{~s}^{-1}$, [lower] Channel maps in the CS $J=2-1$ line with the lowest contour at $0.375 \mathrm{~K} \mathrm{~km} \mathrm{~s}^{-1}$ channel, and subsequent contour intervals increasing by $0.125 \mathrm{~K} \mathrm{~km} \mathrm{~s}^{-1}$. The central $(0,0)$ position of the maps and spectra is that of L1551 IRS5 at RA(2000) $=04^{h} 31^{m} 34^{s} .13, \operatorname{Dec}(2000)=18^{\circ} 08^{\prime} \quad 04^{\prime \prime} .95$.

linewidth toward IRS-5, which we believe is affected by additional dynamics and/or turbulence/outflow activity near L1551 IRS5 which is not modeled here.

\section{OTHER SPECIES IN THE CIRCUMBINARY TORUS}

The integrated $\mathrm{HCN}, \mathrm{CS}, \mathrm{C}^{34} \mathrm{~S}, \mathrm{HCO}^{+}$and $\mathrm{H}^{13} \mathrm{CO}^{+}$ distributions peak close to L1551 IRS-5 (Fridlund et al. 2002), whereas the $\mathrm{CH}_{3} \mathrm{OH}$ map appears as an edgeon torus surrounding the HCN core. The CS and $\mathrm{HCO}^{+}$channel maps show a faint signature of this torus structure, but at low levels not contributing significantly to their integrated emission maps (Fridlund et al in preparation).

Van der Tak et al. (2000) suggest that gas phase pro- 

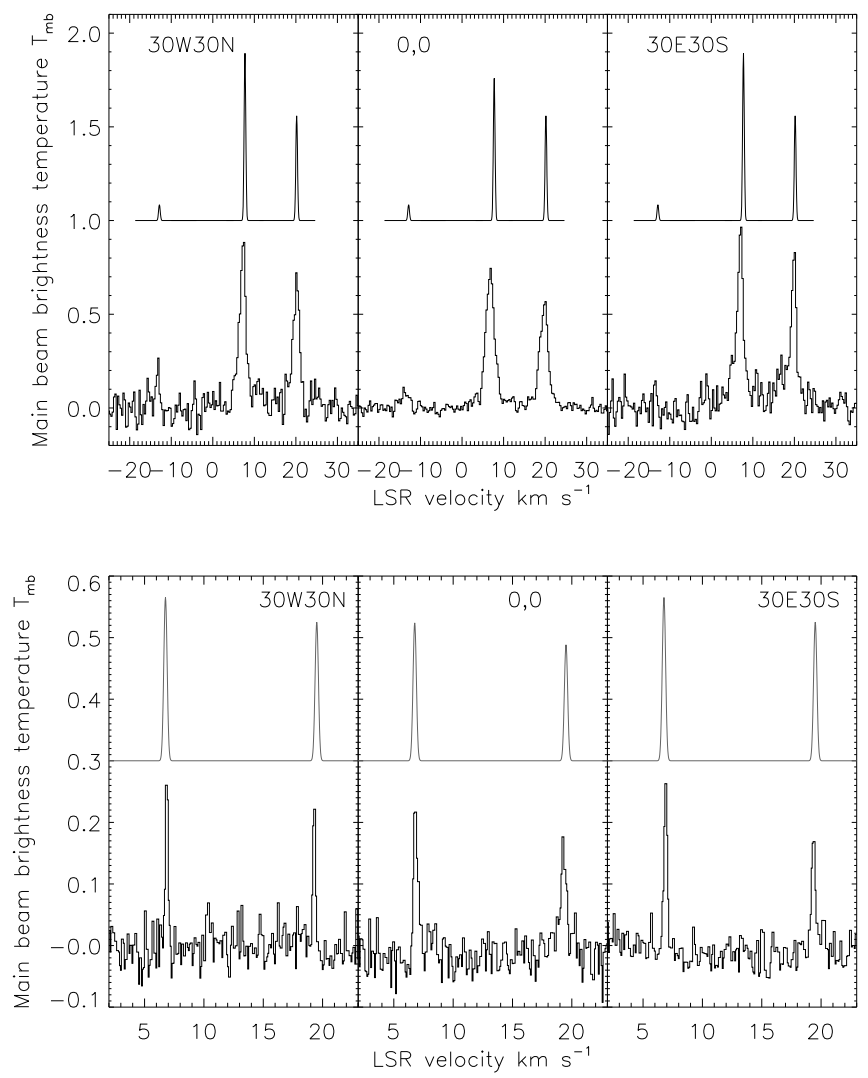

FIG. 3.- $\mathrm{CH}_{3} \mathrm{OH}$ data and model fits for the (left to right) $2_{-1}-1_{-1}(E), 2_{0}-1_{0}(A)$ and $2_{0}-1_{0}(E)$ lines at $96.7394,96.7414$ and $96.7446 \mathrm{GHz}$, and the $5_{-1}-4_{-1}(E)$, and $5_{0}-4_{0}(A)$, lines at 241.7672 and $241.7914 \mathrm{GHz}$ respectively toward the central IRS5 position, and at the two peaks in the $96 \mathrm{GHz} \mathrm{CH}_{3} \mathrm{OH}$ line. The frequency scales assume $\mathrm{v}_{l s r}=6.5 \mathrm{~km} \mathrm{~s}^{-1}$ (Fridlund et al. 2002)

duction of $\mathrm{CH}_{3} \mathrm{OH}$ at temperatures $<100 \mathrm{~K}$ follows the radiative association of $\mathrm{H}_{2} \mathrm{O}$ and $\mathrm{CH}_{3}^{+}$. However, the relative inefficiency of the process would result in low abundances $\sim 10^{-11}$ relative to $\mathrm{H}_{2}$. Recent storage ring measurements (Geppert et al. 2006) of the dissociative recombination of $\mathrm{CH}_{3} \mathrm{OH}_{2}+$ with electrons indicate extensive $\mathrm{C}-\mathrm{O}$ bond fragmentation, emphasising the difficulty for ion-molecule reactions to form $\mathrm{CH}_{3} \mathrm{OH}$, which will preferentially be destroyed via an $\mathrm{H}_{3}^{+}+\mathrm{CH}_{3} \mathrm{OH} \longrightarrow \mathrm{CH}_{3} \mathrm{OH}_{2}^{+}$ $+\mathrm{e}^{+}$reaction. It is increasingly difficult to reconcile observed $\mathrm{CH}_{3} \mathrm{OH}$ abundances with ion-molecule chemical production. Solid phase reactions, including cosmic ray irradiation processes therefore likely are important for $\mathrm{CH}_{3} \mathrm{OH}$ formation on grain surfaces (Wada et al. 2006). Observations in dark clouds, where grain chemistry operates, indicate higher abundances of $\sim 10^{-9}$ (Takakuwa et al. 1998, Turner 1998), and toward hot cores, where $\mathrm{CH}_{3} \mathrm{OH}$ is evaporated off grain surfaces, abundances $\gtrsim$ $10^{-7}$ (Blake et al. 1987) have been reported. Grain temperatures would need to be $\gtrsim 120 \mathrm{~K}$ for methanol to be efficiently released from icy or clathrate mixtures on dust grain surfaces (Blake et al. 1987, Sandford \& Allamandola 1993).

\subsection{Heating of grains}

Our model suggests that gas phase methanol in the envelope is sufficiently cold to rapidly freeze back onto dust
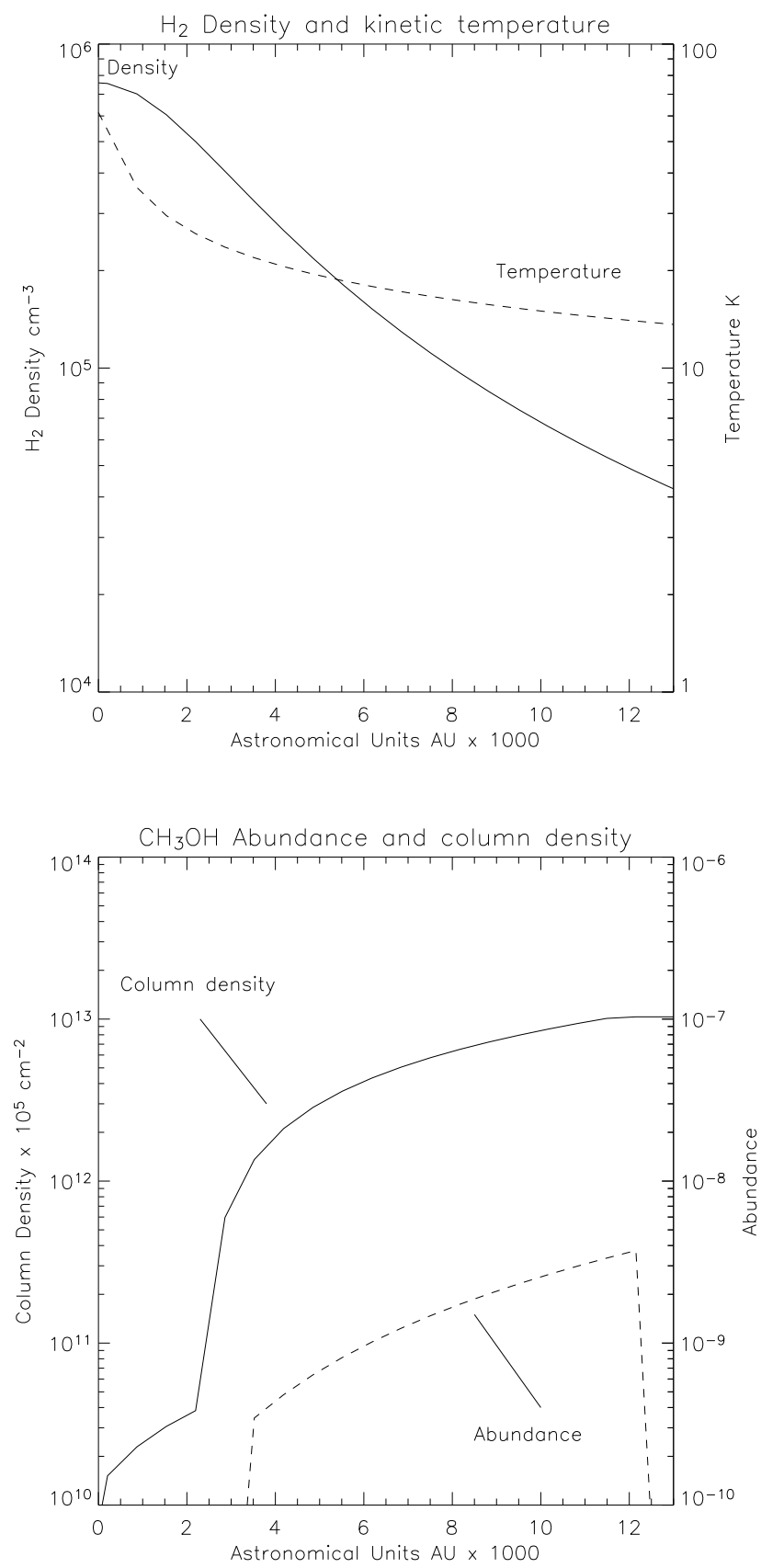

FIG. 4.- (a) Temperature and density from White et al. (2000) used in the $\mathrm{CH}_{3} \mathrm{OH}$ model, (b) abundance and column density estimated from the best fit $\mathrm{CH}_{3} \mathrm{OH}$ model

grains and solid surfaces, which will occur on a timescale $\left(t \sim 3 \times 10^{9} / n(H) \sim 4000\right.$ years, where $n(H)$ is the hydrogen density $\left.\mathrm{cm}^{-3}\right)$. Shocks and direct heating, could re-evaporate the icy mantles, although the liberated $\mathrm{CH}_{3} \mathrm{OH}$ will rapidly cool again. The mid-plane dust temperature, $\mathrm{T}_{\text {dust }}$ due to L1551 IRS5 will be (Fridlund et al. 2002):

$$
\mathrm{T}_{\text {dust }} \approx 38\left(\frac{r}{100 \mathrm{AU}}\right)^{-0.4}\left(\frac{L}{L_{\odot}}\right)^{0.2} \mathrm{~K}
$$

At the $\mathrm{CH}_{3} \mathrm{OH}$ peaks, $\mathrm{T}_{\text {dust }} \sim 13 \mathrm{~K}$ (White et 
al. 2000), which is inadequate for $\mathrm{CH}_{3} \mathrm{OH}$ to be evaporated. Methanol has been observed toward the L1157 molecular disk (Velusamy et al. 2002), and higher mass protostellar cores (van der Tak et al.2000), where direct heating could directly evaporate the $\mathrm{CH}_{3} \mathrm{OH}$ - but it does not appear that this mechanism could drive off significant amounts of $\mathrm{CH}_{3} \mathrm{OH}$ at distance of the L1551 $\mathrm{CH}_{3} \mathrm{OH}$ peaks.

An alternative source of heating is suggested by the presence of an Herbig-Haro jet (HH154) emanating from L1551 IRS5, and terminating in a working surface presently located $\sim 15^{\prime \prime}$ away from the IRS5 binary (Fridlund et al.2005). This jet is one of the few Xray sources associated with low mass stellar jets, and has 4 separate shock interactions - two detected in Xrays (Favata et al.2002, 2006, Bally et al.2003), and two seen in [OIII] $5007 \AA$ and $\mathrm{H} \alpha$ (Fridlund et al. 2005). Assuming a clear line of sight to the surface of the flared disk/envelope/torus surface, the temperatures of icy grains heated by the jet luminosity would be (Hollenbach \& McKee, 1979):

$$
\mathrm{T}_{\text {grain }}=47\left[\frac{J_{U V}}{a_{\mu} \times C_{1}}\right] \mathrm{K}
$$

for ice, where $\mathrm{C}_{1}$ is 2 , the grain radius, $\mathrm{a}_{\mu}$ is assumed to be $0.15 \mu \mathrm{m}$ (Brown, 1990) and the mean intensity is $J_{U V}$. The shock luminosity is then:

$$
F_{S}=5.8 \times 10^{-4} n_{0} v_{s 7}^{3} \quad \mathrm{erg}_{\mathrm{cm}^{-2}} \mathrm{~s}^{-1}
$$

The projected distance of the methanol peaks from the shocks is $\approx 6000 \mathrm{AU}$. Assuming this flux is dominated by X-rays, EUV and FUV emission (Hollenbach \& McKee 1979; Hartigan et al. 1987) $\mathrm{T}_{\text {grain }}$ will reach $100-150 \mathrm{~K}$ which is adequate to liberate $\mathrm{CH}_{3} \mathrm{OH}$. The grains would however rapidly cool again, with the evaporated gas becoming thermally well coupled with the grains. The infall of material would naturally move grains initially at the edge of the torus from the zone exposed to x-rays and EUV/VUV photons to cooler shielded regions of the torus.

The gas phase $\mathrm{CH}_{3} \mathrm{OH}$ abundance in L1551's outer molecular envelope will depend on the balance between several processes including: $a$ ) freeze-out onto dust grains; b) destruction by ion-molecule reactions, since enhanced ion densities will be induced by the x-ray irradiation forming $\mathrm{CH}_{3} \mathrm{OH}$ on a similar timescale to grain surface reactions (Wada et al. 2006); and c) evaporation or release from dust grains following grain heating, or vapourisation by an accretion shock (Velusamy, Langer \& Goldsmith 2002).

To test the importance of $b$ ) above, a chemical model was kindly run for us by Dr S. Viti (Lintott \& Viti 2006 in preparation) for a molecular cloud having solar metallicity and a high (100 x standard) ionization rate, with the same temperature and density to those we infer. This suggests that a high methanol abundance could survive against ion-molecule reactions for at least $10^{4}$ yrs i.e. similar to or greater than the freeze-out timescale. For $c)$, we favor release from heated grains over the accretion shock scenario on the basis of the narrow $\mathrm{CH}_{3} \mathrm{OH}$ linewidths that are observed in the outer L1551 molecular envelope $\left(\sim 0.6 \mathrm{~km} \mathrm{~s}^{-1}\right.$ compared to several $\mathrm{km} \mathrm{s}^{-1}$ in the L1157 inner core), and the distance of the $\mathrm{CH}_{3} \mathrm{OH}$ peaks from L1551 IRS-5. There is no compelling evidence to suggest the $\mathrm{CH}_{3} \mathrm{OH}$ abundance in the outer L1551 torus is significantly influenced by an accretion shock: the narrow $\mathrm{CH}_{3} \mathrm{OH}$ line center lies within $0.1 \mathrm{~km} \mathrm{~s}^{-1}$ of the systemic velocity inferred from many other lines $(\sim$ $6.5 \mathrm{~km} \mathrm{~s}^{-1}-$ Fridlund et al. 2002).

\subsection{Discussion and Conclusions}

Star formation occurs in the dense cores of molecular clouds. Assuming that comet formation occurs as an adjunct to this process, information about the primordial chemical abundances should be locked into the the dust grains and comets, which are the main repositories of primitive material left over from the solar protostellar disc. The molecular inventory of protostellar envelope material has been discussed by White et al. (2003), with $\mathrm{CH}_{3} \mathrm{OH}$ commonly being found in comets (Mumma et al.2003), along with other organic molecules. Our detection of methanol in a region at a similar distance from L1551 IRS5 as that of the Oort Cloud from our own Sun, suggests that the $\mathrm{CH}_{3} \mathrm{OH}$ may be able to accrete to and spread throughout the outer L1551 protostellar envelope, seeding dust grains and primitive bodies with chemical precursor material that could contribute to the synthesis of more complex molecules. This detection of methanol in the L1551 circumbinary torus provides a first glimpse into the initial chemical conditions of matter infalling onto protostellar disks, and future determinations of the abundances of other molecules in the X-ray/EUV illuminated zone could therefore provide a interesting test of the "interstellar" versus "nebular" aspects of cometary and planetesimal chemistry.

In summary: $a)$ we have detected a massive $(0.03$ $\left.M_{\oplus}\right)$, and most likely cold $(\leq 20 \mathrm{~K}$ - based on dust continuum measurements), toroidal shaped ring of methanol surrounding the L1551 IRS5 protobinary system, b) we propose a new mechanism where shock luminosity heating by the jets associated with an externally located x-ray source - these have a direct line of sight to the torus surface could raise the dust temperature sufficiently to liberate $\mathrm{CH}_{3} \mathrm{OH}, c$ ) we suggest that the luminosity from the jet/x-ray source located just above the L1551 torus is able to heat the dust grains sufficiently to liberate a substantial amount of gaseous $\mathrm{CH}_{3} \mathrm{OH}$ in the outer disk of L1551, and $d$ ) we speculate that the methanol released will rapidly freeze back onto solid material in the outer envelope, spreading $\mathrm{CH}_{3} \mathrm{OH}$ to the surfaces of dust grains and primitive bodies - and potentially modifying the primitive surfaces of solid material in L1551's outer molecular envelope. The observations give indications of the initial chemical conditions of matter infalling onto the disks.

We acknowledge discussions with B. Davidson, G. Moriarty-Schieven, V. Ossenkopf, R. Nelson, H. Rickman, F. van der Tak, M. Walmsley, D. Williams and S. Viti, and thank D. Clements and H. Butner for help in obtaining the JCMT service observations, and an anonymous referee for helpful comments. 


\section{REFERENCES}

Bally, J., Feigelson, E., Reipurth, B., 2003, Ap.J., 584, 843

Blake, G.A., Sutton, E.C., Masson, C.R. et al.1987. ApJ, 315, 621 Brown, P.D., 1990, MNRAS, 243, 65

Favata, F., Fridlund, C.V.M., Micela, G. et al. 2002, A\&A, 386, 204.

Favata, F., Giardino, G., Sciortino, S. et al. 2003, A\&A, 403, 187.

Favata, F., Bonito, R., Micela, G. et al. 2006, A\&A, 450, L17.

Fridlund, C.V.M., White, G.J., 1989, A\&A, 223, L13

Fridlund, C.V.M., Liseau, R. 1998, ApJ, 499, L75

Fridlund, C.V.M., Bergman, P., White, G.J. et al. 2002, A\&A, 382, 573.

Fridlund, C.V.M., Liseau, R., Djupvik, A.A. et al. 2005, A\&A, 436, 983

Geppert, W., Hellberg, F. and Osterdahl, F. et al.2006, Faraday Discussions, in press.

Goldsmith, P.F., Langer, W.D. and Velusamy, T. 1999, ApJ, 519, L173

Hartigan, P., Raymond, J., Hartmann, L., 1987, Ap.J., 316, 323

Hollenbach, D., McKee, C.F., 1979, Ap.J.S., 41, 555

Kaifu, N., Hasegawa, T., Morimoto, M. et al. 1984, A\&A, 134, 7

Maret, S., Ceccarelli, C., Tielens, A.G.G.M et al. 2005, A\&A, 442, 527

Moriarty-Schieven, G., Johnstone, D., Bally, J. et al. , 2006, ApJ., 645,357

Müller, H.S.P., Thorwirth, S., Roth, D.A. et al. 2001, A\&A, 370, L49

Mumma, M.J., DiSanti, M.A., Dello Russo, N. et al., 2003, Adv. Space Res, 31, 2563
Parker, N., White,G.J., Williams, P.G. et al. , S.S. 1991, A\&A, 250, 134

Phillips, R.R., Little, L.T. 2000, MNRAS, 317, 179

Pottage, J.T., Flower, D.R., \& Davis, S.L. 2004, MNRAS. 352, 39

Rainey, R., White, G.J., Richardson, K.J. et al. . 1987, A\&A, 179, 237

Sandford, S.A., Allamandola, L.J. 1993, ApJ, 417, 815

Snell, R.L., Loren, R.B., \& Plambeck, R.L. 1980, ApJL, 239, L17

Stocke, J.T., Hartigan, P.M., Strom, S.E. et al., 1988, ApJS, 68, 229

Takakuwa, S., Mikami, H., Saito, M. 1998, ApJ, 501,723

Takakuwa, S., Ohashi, N., Ho, P.T.P., et al. 2004, ApJ, 616, L15

Turner, B.E. 1998, ApJ, 501, 731

van der Tak, F.F.S., van Dishoeck, E.F. Caselli, P. 2000, A\&A, 361,327

Velusamy, T., Langer, W.D. and Goldsmith, P.F. 2002, ApJ., 565, L43

Wada, A., Mochizuki, N \& Hiraoka, K. 2006, ApJ, 644, 300

White, G. J., Liseau, R.L., Men'shchikov, A., et al. 2000, A\&A, 364,741

White, G.J., Thompson, M.A., Fridlund, C.V.M., et al.2003, Proc. Towards Other Earths: DARWIN/TPF and the Search for Extrasolar Terrestrial Planets. Edited by M. Fridlund, T. Henning. ESA SP-539, 653 